\title{
The extremal problems on the inertia of weighted bicyclic graphs*
}

\author{
Shibing Deng, Shuchao Li寸, Feifei Song
}

Faculty of Mathematics and Statistics, Central China Normal University, Wuhan 430079, P.R. China

\begin{abstract}
Let $G_{w}$ be a weighted graph. The number of the positive, negative and zero eigenvalues in the spectrum of $G_{w}$ are called positive inertia index, negative inertia index and nullity of $G_{w}$, and denoted by $i_{+}\left(G_{w}\right), i_{-}\left(G_{w}\right)$, $i_{0}\left(G_{w}\right)$, respectively. In this paper, sharp lower bound on the positive (resp. negative) inertia index of weighted bicyclic graphs of order $n$ with pendant vertices is obtained. Moreover, all the weighted bicyclic graphs of order $n$ with at most two positive, two negative and at least $n-4$ zero eigenvalues are identified, respectively.
\end{abstract}

Keywords: Weighted bicyclic graphs; Adjacency matrix; Inertia

AMS subject classification: 05C50; 15A18

\section{Introduction}

In this paper, we only consider simple weighted graphs on positive weight set. Let $G_{w}$ be a weighted graph with vertex set $\left\{v_{1}, v_{2}, \ldots, v_{n}\right\}$, edge set $E(G) \neq \emptyset$ and $W\left(G_{w}\right)=\left\{w_{j}>0, j=1,2, \ldots,|E(G)|\right\}$. The function $w: E(G) \rightarrow W\left(G_{w}\right)$ is called a weight function of $G_{w}$. It is obvious that each weighted graph corresponds to a weight function. The adjacency matrix of $G_{w}$ on $n$ vertices is defined as the matrix $A\left(G_{w}\right)=\left(a_{i j}\right)$ such that $a_{i j}=$ $w\left(v_{i} v_{j}\right)$ if $v_{i} v_{j} \in E(G)$ and 0 otherwise. The eigenvalues $\lambda_{1}, \lambda_{2}, \ldots, \lambda_{n}$ of $A\left(G_{w}\right)$ are said to be the eigenvalues of the weighted graph $G_{w}$. The inertia of $G_{w}$ is defined to be the triple $\operatorname{In}\left(G_{w}\right)=\left(i_{+}\left(G_{w}\right), i_{-}\left(G_{w}\right), i_{0}\left(G_{w}\right)\right)$, where $i_{+}\left(G_{w}\right), i_{-}\left(G_{w}\right)$ and $i_{0}\left(G_{w}\right)$ are the numbers of the positive, negative and zero eigenvalues of $A\left(G_{w}\right)$ including multiplicities, respectively. $i_{+}\left(G_{w}\right)$ and $i_{-}\left(G_{w}\right)$ are called the positive, negative index of inertia (for short, positive, negative index) of $G_{w}$, respectively. The number $i_{0}\left(G_{w}\right)$ is called the nullity of $G_{w}$. Obviously, $i_{+}\left(G_{w}\right)+i_{-}\left(G_{w}\right)+i_{0}\left(G_{w}\right)=n$.

An induced subgraph of $G_{w}$ is an induced subgraph of $G$ having the same weights with those of $G_{w}$. For an induced weighted subgraph $H_{w}$ of the weighted graph $G_{w}$, let $G_{w}-H_{w}$ be the subgraph obtained from $G_{w}$ by deleting all vertices of $H_{w}$ and all incident edges. We define that the union of $G_{w}^{1}$ and $G_{w}^{2}$, denoted by $G_{w}^{1} \cup G_{w}^{2}$, is the graph with vertex-set $V\left(G_{w}^{1}\right) \cup V\left(G_{w}^{2}\right)$, edge-set $E\left(G_{w}^{1}\right) \cup E\left(G_{w}^{2}\right)$ and the weight of each edge is not changed. A bicyclic graph is a simple connected graph in which the number of edges equals the number of vertices plus 1. A weighted path and a weighted cycle of order $n$ are denoted by $P_{w}^{n}, C_{w}^{n}$, respectively. An isolated vertex is sometime denoted by $K_{1}$.

The study of eigenvalues of graph has been received a lot of attention due to its applications in chemitry (see 2, 1, 10, 15, for details). As we know, if $G$ is a bipartite graph, then $i_{+}(G)=i_{-}(G)=\alpha(G)=\frac{n-i_{0}(G)}{2}$, where $\alpha(G)$ is the matching number of $G$, otherwise, $i_{+}(G), i_{-}(G)$ and $i_{0}(G)$ do dot have this relationship. Gregory et al. 8 studied the subadditivity of the positive, negative indices of inertia and developed certain properties of Hermitian rank which were used to characterize the biclique decomposition number. Gregory et al. 9] investigated the inertia of a partial join of two graphs and established a few relations between inertia and biclique decompositions of partial joins of graphs. Daugherty [3] characterized the inertia of unicyclic graphs in terms of matching number and obtained a linear-time algorithm for computing it. Yu et al. [19] investigated the minimal positive index of

\footnotetext{
*Financially supported by the National Natural Science Foundation of China (Grant Nos. 11271149, 11371062), the Program for New Century Excellent Talents in University (Grant No. NCET-13-0817) and the Special Fund for Basic Scientific Research of Central Colleges (Grant No. CCNU13F020))

${ }^{\dagger}$ Corresponding author.

Email addresses: 750861119@qq.com (S.B. Deng), lscmath@mail.ccnu.edu.cn (S.C. Li), 928046810@qq.com (F.F. Song)
} 
inertia among all unweighted bicyclic graphs of order $n$ with pendants, and characterized the bicyclic graphs with positive index 1 or 2. Very recently, it is interesting to see that Marina et al. [1 studied the inertia set of a signed graph in algebraic approach.

The nullity of unweighted graphs has been studied well in the literature. Tan and Liu [18] gave the nullity set of unicyclic graphs and characterized the unicyclic graphs with maximum nullity. In addition, Nath and Sarma 17. presented another version of characterization of an acyclic or unicyclic graph to be singular. One of the present authors [13] investigated the nullity of graphs with pendant vertices. Fan and Qian [6] characterized the bipartite graphs with the second largest nullity and the regular bipartite graphs with the third largest nullity. Fan and Wang [5] characterized the unicyclic signed graphs of order $n$ with nullity $n-2, n-3, n-4, n-5$, respectively.

Our paper is motivated directly by [4, 11, 13, 16]. On the one hand, Fan et al. [4] studied the nullity of signed bicyclic graph (which is, in fact, the bicyclic graph with edge weight 1 or -1); Li [13] and Hu [11] studied the nullity of unweighted bicyclic graph. On the other hand, Yu et al. [19] characterized all $n$-vertex unweighted bicyclic graphs with positive index 1 or 2 . It is natural and interesting for us to consider the extremal problems on the inertia of weighted bicyclic graphs, which may generalize corresponding results of [4, 11, 13, 19.

This paper is organized as follows: in Section 2, some preliminaries are introduced. In Section 3, we present the lower bound for the positive, negative index of $n$-vertex weighted bicyclic graphs with pendants. In Section 4 , we characterize all $n$-vertex weighted bicyclic graphs without pendant twins having one or two positive (resp. negative) eigenvalues. In Section 5 , we characterize all $n$-vertex weighted bicyclic graphs without pendant twins of rank $2,3,4$.

\section{Preliminaries}

In this section, we list some lemmas which will be used to prove our main results. Suppose $M, N$ are two Hermitian matrices of order $n$, if there exists an invertible matrix $Q$ of order $n$ such that $Q M Q^{*}=N, Q^{*}$ denotes the conjugate transpose of $Q$, then we say that $M$ is congruent to $N$, denoted by $M \cong N$.

Lemma $2.1([12])$. Let $M, N$ be two Hermitian matrices of order $n$ such that $M \cong N$, then $i_{+}(M)=i_{+}(N), i_{-}(M)=$ $i_{-}(N)$ and $i_{0}(M)=i_{0}(N)$.

It is easy to obtain the following result.

Lemma 2.2. Let $G_{w}=G_{w}^{1} \cup G_{w}^{2} \cup \ldots \cup G_{w}^{t}$ be a weighted graph, where $G_{w}^{i}(i=1,2, \ldots, t)$ are connected components of $G_{w}$. Then $i_{+}\left(G_{w}\right)=\sum_{i=1}^{t} i_{+}\left(G_{w}^{i}\right), i_{-}\left(G_{w}\right)=\sum_{i=1}^{t} i_{-}\left(G_{w}^{i}\right)$ and $i_{0}\left(G_{w}\right)=\sum_{i=1}^{t} i_{0}\left(G_{w}^{i}\right)$.

Let $M$ be a Hermitian matrix. We denoted three types of elementary congruence matrix operations (ECMOs) on $M$ as follows:

1. interchanging $i$ th and $j$ th rows of $M$, while interchanging $i$ th and $j$ th columns of $M$;

2. multiplying $i$ th row of $M$ by a non-zero number $k$, while multiplying $i$ th column of $M$ by $k$;

3. adding $i$ th row of $M$ multiplied by a non-zero number $k$ to $j$ th row, while adding $i$ th column of $M$ multiplied by $k$ to $j$ th column.

By Lemma 2.1, the ECMOs do not change the inertia of a Hermitian matrix.

Lemma 2.3 ([19]). Let $M$ be an $n \times n$ Hermitian matrix and $N$ be the Hermitian matrix obtained by bordering $M$ as followings:

$$
N=\left(\begin{array}{cc}
M & y \\
y^{*} & a
\end{array}\right)
$$


where $y$ is a column vector, $y^{*}$ denotes the conjugate transpose of $y$ and $a$ is a real number. Then $i_{+}(N)-1 \leq$ $i_{+}(M) \leq i_{+}(N), i_{-}(M)-1 \leq i_{-}(M) \leq i_{-}(N)$.

By Lemma 2.3 we can get the following result immediately:

Lemma 2.4 ([14]). Let $H_{w}$ be an induced subgraph of $G$. Then $i_{+}\left(H_{w}\right) \leq i_{+}\left(G_{w}\right)$ and $i_{-}\left(H_{w}\right) \leq i_{-}\left(G_{w}\right)$.

Lemma 2.5 ([14]). Let $C_{w}^{n}$ be a weighted cycle of order $n$. Then

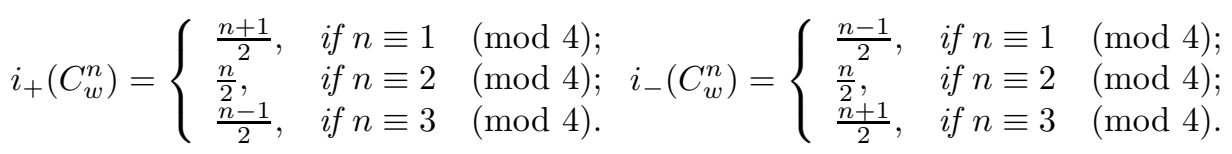

Furthermore, if $n \equiv 0(\bmod 4)$, let $C_{w}^{n}=v_{1} v_{2} \ldots v_{n} v_{1}$ be a weighted cycle of order $n, w\left(v_{i} v_{i+1}\right)=a_{i}(1 \leq i \leq n)$ and let $v_{n+1}=v_{1}$. Then

$$
i_{+}\left(C_{w}^{n}\right)=i_{-}\left(C_{w}^{n}\right)= \begin{cases}\frac{n}{2}-1, & \text { if } \prod_{i=1}^{\frac{n}{2}} a_{2 i-1}=\prod_{i=1}^{\frac{n}{2}} a_{2 i} ; \\ \frac{n}{2}, & \text { otherwise. }\end{cases}
$$

Lemma 2.6 (14]). Let $G_{w}$ be a graph containing a pendant vertex $v$ with its unique neighbor $u$. Then $i_{+}\left(G_{w}\right)=$ $i_{+}\left(G_{w}-u-v\right)+1$ and $i_{-}\left(G_{w}\right)=i_{-}\left(G_{w}-u-v\right)+1$.

The following result is an immediate consequence of Lemma 2.6.

Lemma 2.7. Let $P_{w}^{n}$ be a weighted path of order $n$. Then

$$
i_{+}\left(P_{w}^{n}\right)=i_{-}\left(P_{w}^{n}\right)= \begin{cases}\frac{n-1}{2}, & \text { if } n \text { are odd } \\ \frac{n}{2}, & \text { if } n \text { are even }\end{cases}
$$

Let $u, v$ be two pendant vertices of a weighted graph $G_{w}, u, v$ are called a pendant twin if they have the same neighborhood in $G_{w}$. The following result is an immediate consequence of Lemma 2.6 since $i_{+}\left(K_{1}\right)=i_{-}\left(K_{1}\right)=0$.

Lemma 2.8. If $u, v$ is a pendant twin in a weighted graph $G_{w}$, then $i_{+}\left(G_{w}\right)=i_{+}\left(G_{w}-v\right)=i_{+}\left(G_{w}-u\right)$ and $i_{-}\left(G_{w}\right)=i_{-}\left(G_{w}-v\right)=i_{-}\left(G_{w}-u\right)$.

Let $S_{w}^{k}$ be a weighted star of order $k$ with center $v$ and non-central vertices $v_{1}, \ldots, v_{k-1}$. We can get the following two lemmas by Lemmas 2.4 and 2.6.

Lemma 2.9. Let $G_{w}^{0}$ be a weighted graph of order $n-k$ such that $u \in V\left(G_{w}^{0}\right)$. Let $G_{w}^{1}$ be the graph obtained from $G_{w}^{0}$ and $S_{w}^{k}$ by inserting an edge between $u$ and the center $v$ of $S_{w}^{k}$. Let $G_{w}^{2}=G_{w}^{1}-\left\{v v_{1}, v v_{2}, \ldots, v v_{k-1}\right\}+$ $\left\{u v_{1}, u v_{2}, \ldots, u v_{k-1}\right\}$ where $w\left(u v_{i}\right)=w\left(v v_{i}\right)$. Then $i_{+}\left(G_{w}^{1}\right) \geq i_{+}\left(G_{w}^{2}\right)$ and $i_{-}\left(G_{w}^{1}\right) \geq i_{-}\left(G_{w}^{2}\right)$.

Lemma 2.10. Let $G_{w}^{0}$ be a weighted graph of order $n-l-t$ and $u_{1}, u_{2} \in V\left(G_{0}\right)$. Assume that $G_{w}^{1}$ is the graph obtained from $G_{w}^{0}, S_{w}^{l+1}$ and $S_{w}^{t+1}$ by identifying $u_{1}$ with the center of $S_{w}^{l+1}, u_{2}$ with the center of $S_{w}^{t+1}$,respectively. Let $G_{w}^{2}$ be the graph obtained from $G_{w}^{0}, S_{w}^{l+t+1}$ by identifying $u_{1}$ with the center of $S_{l+t+1}$. Then $i_{+}\left(G_{w}^{1}\right) \geq i_{+}\left(G_{w}^{2}\right)$ and $i_{-}\left(G_{w}^{1}\right) \geq i_{-}\left(G_{w}^{2}\right)$.

Lemma 2.11. Let $G_{w}^{1}$ and $G_{w}^{2}$ be two weighted graphs with $u \in V\left(G_{1}\right)$ and $v \in V\left(G_{2}\right)$. Let $P_{w}^{l}(l \geq 3)$ be a weighted path with two end-vertices $v_{1}, v_{l}$. Let $S_{w}^{l}$ be a weighted star of order $l$ and have the same weight set with $P_{w}^{l}(l \geq 3)$. Let $G_{w}^{\prime}$ be the graph obtained from $G_{w}^{1} \cup G_{w}^{2} \cup P_{w}^{l}$ by identifying $u$ with $v_{1}$ and $v$ with $v_{l}$, respectively. Let $G_{w}^{\prime \prime}$ be the graph obtained from $G_{w}^{1} \cup G_{w}^{2}$ by identifying $u, v$ with the center of $S_{w}^{l}$. Then $i_{+}\left(G_{w}^{\prime}\right) \geq i_{+}\left(G_{w}^{\prime \prime}\right)$ and $i_{-}\left(G_{w}^{\prime}\right) \geq i_{-}\left(G_{w}^{\prime \prime}\right)$. 
Proof. In view of Lemma 2.6, we have

$$
i_{+}\left(G_{w}^{\prime \prime}\right)=1+i_{+}\left(G_{w}^{1}-u\right)+i_{+}\left(G_{w}^{2}-v\right)
$$

Note that $\left(G_{w}^{1}-u\right) \bigcup\left(G_{w}^{2}-v\right) \bigcup P_{w}^{l-1}$ is an induced subgraph of $G_{w}^{\prime}$. In light of Lemma 2.3 , it follows that

$$
i_{+}\left(G_{w}^{1}-v\right)+i_{+}\left(G_{w}^{2}-u\right)+i_{+}\left(P_{w}^{l-1}\right) \leq i_{+}\left(G_{w}^{\prime}\right) .
$$

By Lemma $2.7, i_{+}\left(P_{w}^{l-1}\right) \geq 1(l \geq 3)$, therefore $i_{+}\left(G_{w}^{\prime}\right) \geq i_{+}\left(G_{w}^{\prime \prime}\right)$. Similarly, $i_{-}\left(G_{w}^{\prime}\right) \geq i_{-}\left(G_{w}^{\prime \prime}\right)$, as desired.

\section{The minimal positive (negative) index of inertia of weighted bicyclic graphs}

Let $G$ be a bicyclic graph. The base of $G$, denoted by $\chi(G)$, is the unique bicyclic subgraph of $G$ containing no pendant vertices. Thus $G$ can be obtained from $\chi(G)$ by attaching trees to some vertices of $\chi(G)$. Let $C^{p}(p \geq 3)$ and $C^{q}(q \geq 3)$ be two vertex-disjoint cycles of length $p, q$ and $P_{l}=v_{1} v_{2} \ldots v_{l}(l \geq 1)$ be a path of length $l-1$. Assume that $v \in V\left(C^{p}\right)$ and $u \in V\left(C^{q}\right)$. Let $\infty(p, l, q)$ be the graph obtained from $C^{p}, C^{q}$ and $P_{l}$ by identifying $v$ with $v_{1}, u$ with $v_{l}$. Let $P_{p+2}, P_{l+2}, P_{q+2}$ be three paths with $\min \{p, l, q\} \geq 0$ and at most one of $p, l, q$ is 0 . Let $\theta(p, l, q)$ be the graph obtained from $P_{p+2}, P_{l+2}$ and $P_{q+2}$ by identifying the three initial vertices and terminal vertices. The weighted graphs $\infty(p, l, q)_{w}$ and $\theta(p, l, q)_{w}$ are depicted in Fig. 1, where the number on each edge denotes its weight. In what follows in our context, we always assume that the weight for each edge of $\infty(p, l, q)_{w}$ (resp. $\left.\theta(p, l, q)_{w}\right)$ are as shown in Fig. 1.
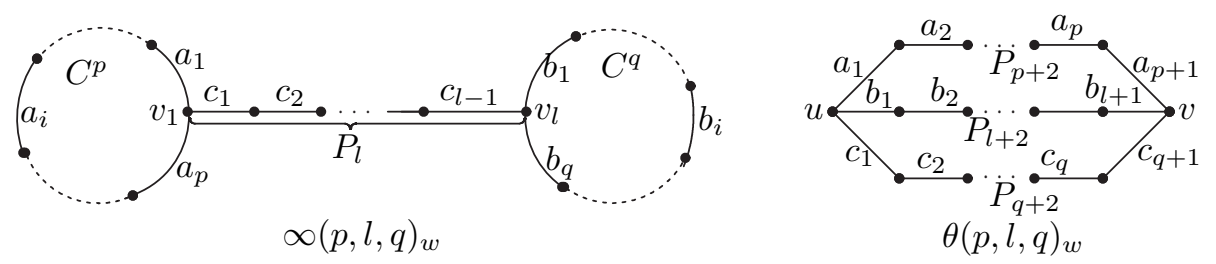

Figure 1: Weighted graphs $\infty(p, l, q)_{w}$ and $\theta(p, l, q)_{w}$.

As we know, the connected bicyclic graphs can be partitioned into two classes: one class of bicyclic graphs contain $\infty(p, l, q)$ as its basis and the other class of bicyclic graphs contain $\theta(p, l, q)$ as its basis. We call bicyclic graph $G$ an $\infty$-graph if $G$ contains some $\infty(p, l, q)$ as its basis and a $\theta$-graph if $G$ contains some $\theta(p, l, q)$ as its basis. We denote by $\mathscr{B}$ (resp. $\mathscr{B}_{p}$ ) the set of all weighted bicyclic graphs (resp. weighted bicyclic graphs with pendants) of order $n$. Let $\chi\left(G_{w}\right)$ be the base of $G_{w}$, by Lemma 2.6, there is no correlation between the inertia index of $G_{w}$ and the weighted set of $G_{w}-\chi\left(G_{w}\right)$. Hence, in order to determine $\operatorname{In}\left(G_{w}\right)$, it suffices to consider the weight of $\chi\left(G_{w}\right)$ in what follows.

Theorem 3.1. Let $G_{w} \in \mathscr{B}_{p}$ and contain $\infty(p, l, q)$ as its base. Then

$$
i_{+}\left(G_{w}\right) \geq \begin{cases}\frac{p+q}{2}, & \text { if } p, q \text { are odd } \\ \frac{p+q}{2}-1, & \text { if } p, q \text { are even } \\ \frac{p+q-1}{2}, & \text { otherwise. }\end{cases}
$$

This bound is sharp.

Proof. For a weighted $\infty$-graph, let $u$ be the common vertex of $C^{p}$ and $C^{q}$ in $\infty(p, 1, q)$. Let $G^{*}$ be the bicyclic graph obtained by attaching $n-p-q+1(n \geq p+q)$ pendants to $u$ (see Fig. 2) and let $G_{w}^{*}$ denote the weighted graph with $G^{*}$ as its underlying graph. 

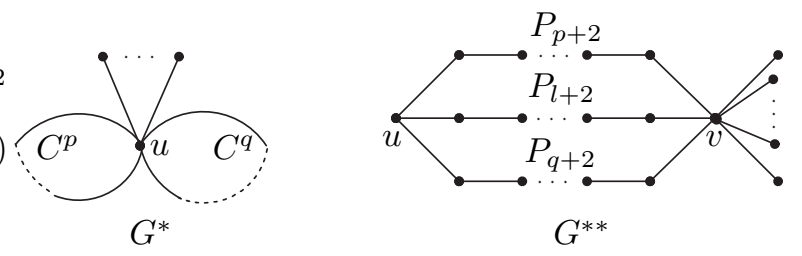

Figure 2: Graphs $G^{*}$ and $G^{* *}$.

Let $\mathscr{S}_{1}(n)$ be the set of all $n$-vertex weighted bicyclic graphs whose underlying graph is obtained from $\infty(p, 1, q)$ by attaching $n-p-q+1(n \geq p+q)$ pendants to a vertex, different from $u$ of $C^{p}$ or $C^{q}$.

Let $\mathscr{S}_{2}(n)$ be the set of all $n$-vertex weighted bicyclic graphs whose underlying graph is obtained from $\infty(p, 2, q)$ by attaching $n-p-q(n \geq p+q+1)$ pendants to the vertex $u$ of $C^{p}$ or $C^{q}$.

In view of Lemma 2.6 , we have

$$
i_{+}\left(G_{w}^{*}\right)=1+i_{+}\left(P_{w}^{p-1}\right)+i_{+}\left(P_{w}^{q-1}\right) .
$$

First we are to show that $i+\left(G_{w}^{*}\right) \leq i_{+}\left(G_{w}\right)$ for any $G_{w} \in \mathscr{S}_{1}(n) \bigcup \mathscr{S}_{2}(n)$. In fact, if $G_{w} \in \mathscr{S}_{1}(n)$, without loss of generality, we suppose all the pendant vertices are attached at $C^{p}$. Then by Lemma 2.6, we have

$$
\begin{aligned}
i_{+}\left(G_{w}\right) & =1+ \begin{cases}\frac{p-1}{2}+i_{+}\left(P_{w}^{q-1}\right), & \text { if } p \text { is odd; } \\
\frac{p-2}{2}+i_{+}\left(C_{w}^{q}\right) \text { or, } i_{+}\left(P_{w}^{q-1}\right), & \text { if } p \text { is even. }\end{cases} \\
& =1+i_{+}\left(P_{w}^{p-1}\right)+ \begin{cases}i_{+}\left(P_{w}^{q-1}\right), & \text { if } p \text { is odd } \\
i_{+}\left(C_{w}^{q}\right) \text { or }, i_{+}\left(P_{w}^{q-1}\right), & \text { if } p \text { is even. }\end{cases}
\end{aligned}
$$

By Lemma $2.4, i_{+}\left(C_{w}^{q}\right) \geq i_{+}\left(P_{w}^{q-1}\right)$. Hence, in view of (3.1) and (3.2) we have $i_{+}\left(G_{w}^{*}\right) \leq i_{+}\left(G_{w}\right)$.

If $G_{w} \in \mathscr{S}_{2}(n)$, without loss of generality, we suppose all the pendant vertices are attached at $C^{p}$. Then by Lemma 2.6 we have

$$
\begin{aligned}
i_{+}\left(G_{w}\right) & =1+ \begin{cases}\frac{p-1}{2}+i_{+}\left(C_{w}^{q}\right), & \text { if } p \text { is odd } \\
\frac{p-2}{2}+i_{+}\left(C_{w}^{q}\right) \text { or } i_{+}\left(G_{w}^{\prime}\right), & \text { if } p \text { is even. }\end{cases} \\
& =1+i_{+}\left(P_{w}^{p-1}\right)+ \begin{cases}i_{+}\left(C_{w}^{q}\right), & \text { if } p \text { is odd } \\
i_{+}\left(C_{w}^{q}\right) \text { or } i_{+}\left(G_{w}^{\prime}\right), & \text { if } p \text { is even, }\end{cases}
\end{aligned}
$$

where $G_{w}^{\prime}$ is a graph obtained by attaching a pendant vertex to a vertex of $C^{q}$. Note that $i_{+}\left(C_{w}^{q}\right) \geq i_{+}\left(P_{w}^{q-1}\right)$ and $i_{+}\left(G_{w}^{\prime}\right) \geq i_{+}\left(P_{w}^{q-1}\right)$ from Lemma 2.4. Hence, in view of (3.1) and (3.3) we have $i_{+}\left(G_{w}^{*}\right) \leq i_{+}\left(G_{w}\right)$.

From Lemmas 2.9, 2.10 and 2.11, $G_{w}^{*}$ attains the minimal positive index among all $n$-vertex weighted bicyclic graphs with pendant vertices containing two edge disjoint weighted cycles $C_{w}^{p}$ and $C_{w}^{q}$.

Similarly, we can have the following theorem:

Theorem 3.2. Let $G_{w} \in \mathscr{B}_{p}$ and contain $\infty(p, l, q)$ as its base. Then

$$
i_{-}\left(G_{w}\right) \geq \begin{cases}\frac{p+q}{2}, & \text { if } p, q \text { are odd; } \\ \frac{p+q}{2}-1, & \text { if } p, q \text { are even; } \\ \frac{p+q-1}{2}, & \text { otherwise. }\end{cases}
$$

This bound is sharp.

By Theorems 3.1 and 3.2, it follows that 
Theorem 3.3. Let $G_{w}$ be a weighted $\infty$-graph of order $n$ with pendant vertices, then $i_{+}\left(G_{w}\right) \geq 3, i_{-}\left(G_{w}\right) \geq 3$ and $i_{0}\left(G_{w}\right) \leq n-6$.

Theorem 3.4. Let $G_{w} \in \mathscr{B}_{p}$ and contain $\theta(p, l, q)$ as its base $(n \geq p+q+l+3)$. If plq $\neq 0$, then

$$
i_{+}\left(G_{w}\right) \geq \begin{cases}1+\frac{p+q+l}{2}, & \text { is } p+q+l \text { is even } \\ \frac{p+q+l}{2}, & \text { if } p, q, l \text { are odd; } \\ 1+\frac{p+q+l+1}{2}, & \text { otherwise. }\end{cases}
$$

This bound is sharp.

Proof. Let $u, v$ be two vertices in $\theta(p, l, q)$ (see Fig. 1) and $\mathscr{S}_{3}(n)(n \geq p+q+l+3)$ be the set of all $n$-vertex weighted bicyclic graphs with $n-p-q-l-2$ pendant vertices attached to a vertex, different from $u$ and $v$ of $\theta(p, l, q)$. Let $G^{* *}$ be the bicyclic graph with $n-p-q-l-2(n \geq p+q+l+3)$ pendant vertices attached to $v$ in $\theta(p, l, q)$ and let $G_{w}^{* *}$ denote the weighted graph with $G^{* *}$ as its underlying graph, where $G^{* *}$ is depicted in Fig. 2. We will verify that $i_{+}\left(G_{w}^{* *}\right) \leq i_{+}\left(G_{w}\right)$ for any $G_{w} \in \mathscr{S}_{3}(n)$.

For any $G_{w} \in \mathscr{S}_{3}(n)$, without loss of generality, assume that $n-p-q-l-2$ pendant vertices are attached to a vertex of $P_{p+2}-u-v$ in $G_{w}$. By Lemma 2.6, we have

$$
\begin{aligned}
i_{+}\left(G_{w}\right) & = \begin{cases}1+\frac{p}{2}+i_{+}\left(P_{w}^{l+q+1}\right), & \text { if } p \text { is even; } \\
1+\frac{p-1}{2}+i_{+}\left(C_{w}^{l+q+2}\right), \text { or } 1+\frac{p+1}{2}+i_{+}\left(P_{w}^{q}\right)+i_{+}\left(P_{w}^{l}\right), & \text { if } p \text { is odd. }\end{cases} \\
i_{+}\left(G_{w}^{* *}\right) & = \begin{cases}1+\frac{p}{2}+i_{+}\left(P_{w}^{l+q+1}\right), & \text { if } p \text { is even; } \\
1+\frac{p+1}{2}+i_{+}\left(P_{w}^{q}\right)+i_{+}\left(P_{w}^{l}\right), & \text { if } p \text { is odd. }\end{cases}
\end{aligned}
$$

Note that $i_{+}\left(C_{w}^{q+l+2}\right) \geq i_{+}\left(P_{w}^{q}\right)+i_{+}\left(P_{w}^{l}\right)+1$ from Lemma 2.3 , hence we have $i_{+}\left(G_{w}^{* *}\right) \leq i_{+}\left(G_{w}\right)$.

By Lemmas 2.9, 2.10 and 2.11, $G_{w}^{* *}$ attains the minimal positive index among all $n$-vertex weighted bicyclic graphs with pendant vertices containing $\theta(p, l, q)$ as its base, $n \geq p+q+l+3$.

Similarly, we can have the following theorem:

Theorem 3.5. Let $G_{w} \in \mathscr{B}_{p}$ and contain $\theta(p, l, q)$ as its base $(n \geq p+q+l+3)$. If plq $\neq 0$, then

$$
i_{-}\left(G_{w}\right) \geq \begin{cases}1+\frac{p+q+l}{2}, & \text { is } p+q+l \text { is even; } \\ \frac{p+q+l}{2}, & \text { if } p, q, l \text { are odd; } \\ 1+\frac{p+q+l+1}{2}, & \text { otherwise. }\end{cases}
$$

This bound is sharp.

Next we consider the special case that one of $p, l, q$ is zero, Without loss of generality, we may assume $l=0$. By a similar discussion as in the proof of Theorem 3.3, we can get the following result.

Theorem 3.6. Let $G_{w} \in \mathscr{B}_{p}$ and contain $\theta(p, 0, q)$ as its base $(n \geq p+q+l+3)$. Then

$$
i_{+}\left(G_{w}\right)=i_{-}\left(G_{w}\right) \geq \begin{cases}1+\frac{p+q}{2}, & \text { if } p+q \text { is even } ; \\ 1+\frac{p+q+1}{2}, & \text { otherwise. }\end{cases}
$$

This bound is sharp.

By Theorems 3.5 and 3.6 we have

Theorem 3.7. Let $G_{w}$ be a weighted $\theta$-graph of order $n$ with pendant vertices. Then $i_{+}\left(G_{w}\right) \geq 2, i_{-}\left(G_{w}\right) \geq 2$ and $i_{0}\left(G_{w}\right) \leq n-4$. 


\section{Characterization of weighted bicyclic graphs with small positive (negative) indices}

In this section we characterize the extremal weighted bicyclic graphs with positive (resp. negative) indices 1, 2.

Theorem 4.1. Let $G_{w} \in \mathscr{B}$. Then $i_{+}\left(G_{w}\right)=1$ if and only if $G_{w}$ is one of the following graphs: the weighted graph $\theta(1,1,1)_{w}$ with weighted condition $c_{1} a_{2}=a_{1} c_{2}$ and $a_{2} b_{1}=a_{1} b_{2}$; the weighted graph $\theta(1,0,1)_{w}$ with weighted condition $a_{2} c_{1}=a_{1} c_{2}$.

Proof. By Theorems 3.3 and 3.7, it suffices to consider the case that the weighted bicyclic graphs of order $n$ without pendant vertices. If $G_{w}$ is a $\infty$-graph, it contains $P_{w}^{2} \cup P_{w}^{2}$ as an induced subgraph, hence $i_{+}\left(G_{w}\right) \geq$ $i_{+}\left(P_{w}^{2} \cup P_{w}^{2}\right)=2$. Then we just need to consider the case that $G_{w}$ is a $\theta$-graph. Without loss of generality, we assume that $l \leq p \leq q$.

If $l=0$, then we have $p+q+1 \leq 3$, otherwise it contains $P_{w}^{4}$ as an induced subgraph and by Lemma 2.7, $i_{+}\left(P_{w}^{4}\right)=2$. Noted that $p+q \geq 2$, then the underlying graph of $G_{w}$ must be $\theta(1,0,1)$. Applying ECMOs to $A\left(G_{w}\right)$ yields $i_{+}\left(G_{w}\right)=1$ if and only if the weight of $G_{w}$ satisfies $a_{2} c_{1}=a_{1} c_{2}$.

If $l>0$, then we have $p+q+2 \leq 4$, otherwise it contains $C_{w}^{k}$ as an induced subgraph and $i_{+}\left(C_{w}^{k}\right) \geq 3$, where $k \geq 5$. Noted that $p+q \geq 2$, then the underlying graph of $G_{w}$ must be $\theta(1,1,1)$. Applying ECMOs to $A\left(G_{w}\right)$ yields $i_{+}\left(G_{w}\right)=1$ if and only if the weight of $G_{w}$ satisfies $a_{2} c_{1}=a_{1} c_{2}$ and $a_{2} b_{1}=a_{1} b_{2}$.

Similarly, we have the following theorem:

Theorem 4.2. Let $G_{w} \in \mathscr{B}$. Then $i_{-}\left(G_{w}\right)=1$ if and only if $G_{w}$ is the weighted graph $\theta(1,1,1)_{w}$ with weighted condition $c_{1} a_{2}=a_{1} c_{2}$ and $a_{2} b_{1}=a_{1} b_{2}$.

Table 1: The weighted condition for each $G_{w} \in \mathscr{B} \backslash \mathscr{B}_{p}$ satisfying $i_{+}\left(G_{w}\right)=2$.

\begin{tabular}{|c|c||c|c|}
\hline weighted graph $G_{w}$ & weighted conditions of $G_{w}$ & weighted graph $G$ & weighted conditions of $G_{w}$ \\
\hline$\infty(3,1,3)_{w}$ & & $\theta(1,1,1)_{w}$ & $a_{2} b_{1} \neq a_{1} b_{2}$, or $a_{2} c_{1} \neq a_{1} c_{2}$ \\
\hline$\infty(3,2,3)_{w}$ & $4 a_{1} a_{3} b_{1} b_{3}-a_{2} b_{2} c_{1}^{2} \geq 0$ & $\theta(1,0,1)_{w}$ & $a_{2} c_{1} \neq a_{1} c_{2}$ \\
\hline$\infty(3,1,4)_{w}$ & $b_{1} b_{3}=b_{2} b_{4}$ & $\theta(1,0,2)_{w}$ & $a_{1} b_{2} \geq c_{1} c_{3}$ \\
\hline$\infty(4,1,4)_{w}$ & $a_{1} a_{3}=a_{2} a_{4}, b_{1} b_{3}=b_{2} b_{4}$ & $\theta(2,0,2)_{w}$ & $a_{2} b_{1} c_{2}=a_{1} a_{3} c_{2}+a_{2} c_{1} c_{3}$ \\
\hline
\end{tabular}

Theorem 4.3. Let $G_{w} \in \mathscr{B} \backslash \mathscr{B}_{p}$, then $i_{+}\left(G_{w}\right)=2$ if and only if $G_{w} \cong \infty(3,1,3)_{w}, \infty(3,2,3)_{w}, \infty(3,1,4)_{w}$, $\infty(4,1,4)_{w}, \theta(1,1,1)_{w}, \theta(1,0,1)_{w}, \theta(1,0,2)_{w}$, or $\theta(2,0,2)_{w}$ and the corresponding weighted conditions are as shown in Table 1, where the empty cell means there is no correlation between the inertia index of $G_{w}$ and its weight set.

Proof. We distinguish the following two possible cases to prove our results.

Case $1 G_{w}$ is a weighted $\infty$-graph.

Note that if $G_{w}$ contains $P_{w}^{6}$ as an induced subgraph, then $i_{+}\left(G_{w}\right) \geq 3$. Hence, it suffices to consider that $p+l+q-4 \leq 5$, i.e., $p+l+q \leq 9$. Note that $p+l+q \geq 7$, hence $7 \leq p+l+q \leq 9$.

If $p+l+q=7$, then $G_{w}$ must be $\infty(3,1,3)_{w}$. Applying the ECMOs to $A\left(G_{w}\right)$, we have $i_{+}\left(G_{w}\right)=2$ and the positive index of $G_{w}$ is independent of its weights. 
If $p+l+q=8$, then $G_{w} \cong \infty(3,2,3)_{w}$ or, $\infty(3,1,4)_{w}$. Applying the ECMOs to $A\left(G_{w}\right)$, if $G_{w} \cong \infty(3,2,3)_{w}$, then we have $i_{+}\left(G_{w}\right)=2$ if and only if the weight of $G_{w}$ satisfies $4 a_{1} a_{3} b_{1} b_{3}-a_{2} b_{2} c_{1}^{2} \geq 0$; if $G_{w} \cong \infty(3,1,4)_{w}$, then we have $i_{+}\left(G_{w}\right)=2$ if and only if the weight of $G_{w}$ satisfies $b_{1} b_{3}=b_{2} b_{4}$.

If $p+l+q=9$, then $G_{w} \cong \infty(3,3,3)_{w}, \infty(3,2,4)_{w}, w \infty(3,1,5)$ or, $\infty(4,1,4)_{w}$. Applying the ECMOs to $A\left(G_{w}\right)$, if $G_{w} \cong \infty(4,1,4)_{w}$, then we have $i_{+}\left(G_{w}\right)=2$ if and only if the weight of $G_{w}$ satisfies $a_{1} a_{3}=a_{2} a_{4}$ and $b_{1} b_{3}=b_{2} b_{4}$; if $G_{w} \cong \infty(3,3,3)_{w}, \infty(3,2,4)_{w}$ or $\infty(3,1,5)_{w}$, then $G_{w}$ contains $H_{w}$ as its induced subgraph, where the underlying graph of $H_{w}$ is depicted in Fig. 3. By Lemma 2.6, $i_{+}\left(G_{w}\right) \geqslant i_{+}\left(H_{w}\right) \geq 3$.

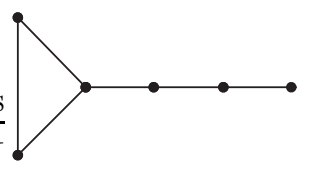

Figure 3: The underlying graph of $H_{w}$.

Case $2 G_{w}$ is a weighted $\theta$-graph. In this case, we assume, without loss of generality, that $l \leq p \leq q$. By Lemmas 2.5 and 2.7, we have $i_{+}\left(P_{w}^{6}\right)=3$ and $i_{+}\left(C_{w}^{k}\right) \geq 3, k \geq 5$. Hence, it suffices to consider that $G_{w}$ does not contain $P_{w}^{6}$ or $C_{w}^{k}$ as an induced subgraph, $k \geq 5$.

First consider $l>0$. In this subcase, we have $p+q+2 \leq 4$, otherwise $G_{w}$ contains $C_{w}^{k}$ as an induced subgraph with $k \geq 5$. Hence, $i_{+}\left(G_{w}\right) \geq i_{+}\left(C_{w}^{k}\right) \geq 3$. It is routine to check that $p+q \geq 2$, hence $p+q=2$, which implies the underlying graph of $G_{w}$ must be $\theta(1,1,1)$. Applying the ECMOs to $A\left(G_{w}\right)$ yields $i_{+}\left(G_{w}\right)=2$ if and only if the weight of $G_{w}$ satisfies $a_{2} b_{1} \neq a_{1} b_{2}$ or, $a_{2} c_{1} \neq a_{1} c_{2}$.

Now consider $l=0$. In this subcase, we have $p+q+1 \leq 5$; otherwise $G_{w}$ contains $P_{w}^{6}$ as an induced subgraph. Note that $p+q \geq 2$, hence $2 \leq p+q \leq 4$.

If $p+q=2$, then $G_{w} \cong \theta(1,0,1)_{w}$. Applying ECMOs to $A\left(G_{w}\right)$ yields $i_{+}\left(G_{w}\right)=2$ if and only if the weight of $G_{w}$ satisfies $a_{2} c_{1} \neq a_{1} c_{2}$. If $p+q=3$, then $G_{w} \cong \theta(1,0,2)_{w}$. Applying ECMOs to $A\left(G_{w}\right)$ yields $i_{+}\left(G_{w}\right)=2$ if and only if the weight of $G_{w}$ satisfies $a_{1} b_{2} \geq c_{1} c_{3}$. If $p+q=4, G_{w} \cong \theta(1,0,3)_{w}$ or, $\theta(2,0,2)_{w}$. If $G_{w} \cong \theta(2,0,2)_{w}$, then applying ECMOs to $A\left(G_{w}\right)$ yields $i_{+}\left(G_{w}\right)=2$ if and only if the weight of $G_{w}$ satisfies $a_{2} b_{1} c_{2}-a_{1} a_{3} c_{2}-a_{2} c_{1} c_{3}=0$. If $G_{w} \cong \theta(1,0,3)_{w}$, then applying ECMOs to $A\left(G_{w}\right)$ yields $i_{+}\left(G_{w}\right)=3$ and the positive index of $G_{w}$ is independent of the weights.

Table 2: The weighted condition for each $G_{w} \in \mathscr{B}_{p}$ but no pendant twins and satisfying $i_{+}\left(G_{w}\right)=2$.

\begin{tabular}{|c|c||c|c|}
\hline weighted graph $G_{w}$ & weighted conditions of $G_{w}$ & weighted graph $G_{w}$ & weighted conditions of $G_{w}$ \\
\hline$G_{w}^{1}, G_{w}^{3}, G_{w}^{6}, G_{w}^{7}, G_{w}^{8}$ & & $G_{w}^{9}, G_{w}^{10}$ & $a_{1} c_{2}=a_{2} c_{1}$ \\
\hline$G_{w}^{2}$ & $a_{1} b_{2}=a_{2} b_{1}$ & $G_{w}^{11}$ & $a_{1} a_{3}=a_{2} b_{1}$ \\
\hline$G_{w}^{4}, G_{w}^{5}$ & $a_{1} b_{2}=a_{2} b_{1}, a_{1} c_{2}=a_{2} c_{1}$ & & \\
\hline
\end{tabular}

In what follows, we shall characterize all weighted bicyclic graphs with pendants having two positive eigenvalues.

Theorem 4.4. Let $G_{w} \in \mathscr{B}_{p}$ but no pendant twins. Then $i_{+}\left(G_{w}\right)=2$ if and only if $G_{w} \cong G_{w}^{1}, G_{w}^{2}, \ldots, G_{w}^{10}$ or, $G_{w}^{11}$ and the corresponding weighted conditions are as shown in Table 2, where the underlying graphs of $G_{w}^{1}, G_{w}^{2}, \ldots, G_{w}^{10}, G_{w}^{11}$ are depicted in Fig. 4 and the empty cell in Table 2 means there is no correlation between the inertia index of $G_{w}$ and its weight set. 


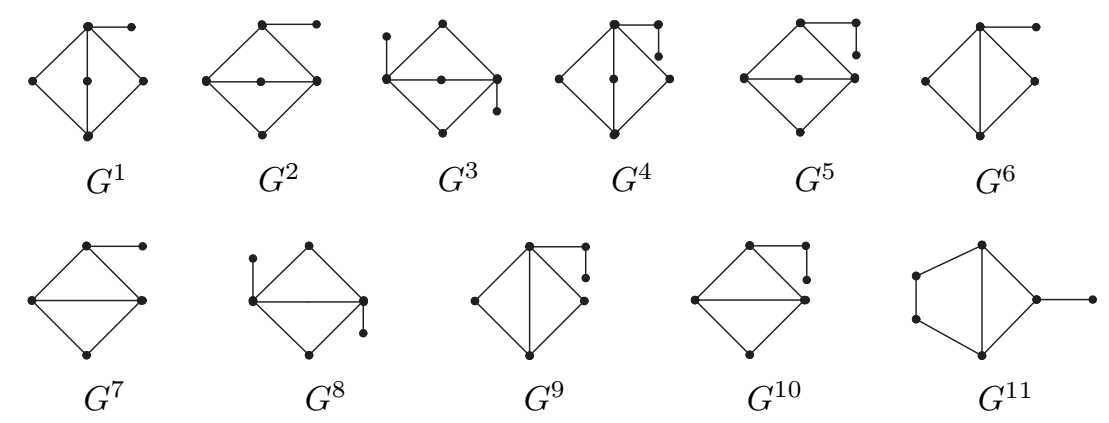

Figure 4: Graphs $G^{1}, G^{2}, \ldots, G^{11}$.
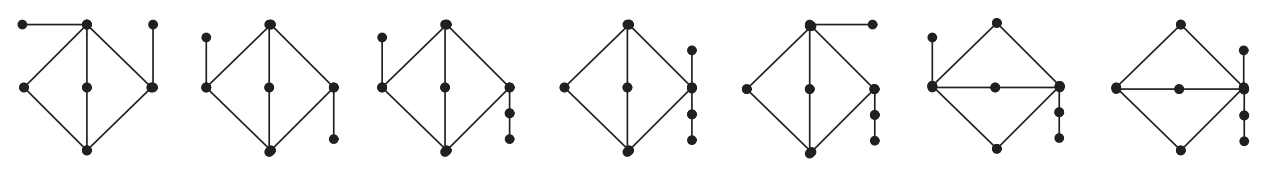

$G^{12}$
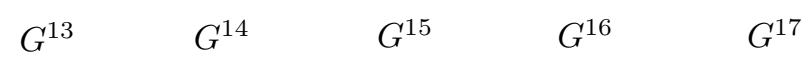

$G^{18}$
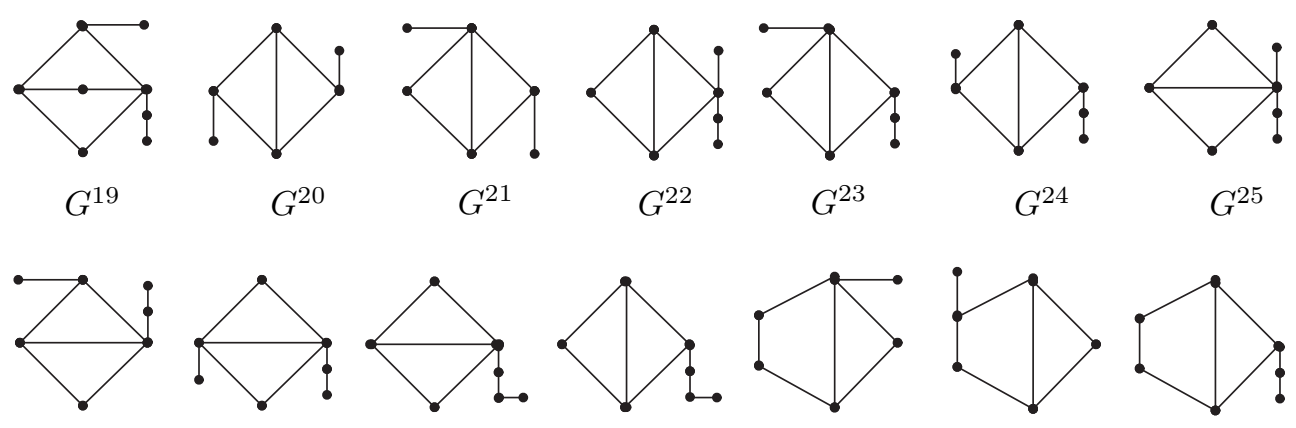

$G^{26}$
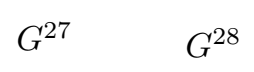

$G^{29}$

$G^{31}$

$G^{32}$
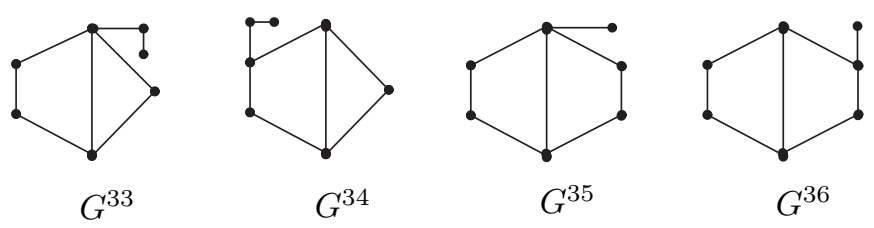

Figure 5: Graphs $G^{12}, G^{13}, \ldots, G^{36}$.

Proof. By Lemmas 2.5, 2.6 and applying the ECMOs, it is routine to check that $i_{+}\left(G_{w}^{i}\right)=2, i=1, \ldots, 11$, and the weight condition for $G_{w}^{i}$ is listed in Table 2. Furthermore, $i_{+}\left(G_{w}^{j}\right) \geq 3$ holds for any weighted condition, $j=12, \ldots, 36$. Here the underlying graphs of $G_{w}^{1}, \ldots, G_{w}^{11}$ are depicted in Fig. 4 , while those of $G_{w}^{12}, \ldots, G_{w}^{36}$ are depicted in Fig. 5 .

Let $\mathcal{H}=G-\chi(G)$ and denote by $v(\mathcal{H})$ the number of vertices of $\mathcal{H}$ in what follows. Note that if $i_{+}\left(G_{w}\right)=2$, by Theorem 3.3, $G_{w}$ must be a weighted $\theta$-graph and by Lemma 2.4 , we have $i_{+}\left(\chi\left(G_{w}\right)\right) \leq 2$. Hence, in view of Theorems 4.1 and 4.3 , we have $\chi(G) \in\{\theta(1,1,1), \theta(1,0,1), \theta(1,0,2), \theta(2,0,2)\}$.

First, we characterize all graphs $G_{w}$ with $\theta(1,1,1)$ as its base satisfying $i_{+}\left(G_{w}\right)=2$ according to the following two possible cases.

Case 1. $\mathcal{H}$ is a collection of isolated vertices. 
If $v(\mathcal{H})=1, G_{w}$ must be $G_{w}^{1}$ or $G_{w}^{2}$. By Lemmas 2.5 and 2.6, we have $i_{+}\left(G_{w}^{2}\right)=2$ if and only if the weight of $G_{w}$ satisfies $a_{1} b_{2}=a_{2} b_{1}$. It is routine to check that $i_{+}\left(G_{w}^{1}\right)=2$.

If $v(\mathcal{H})=2, G_{w}$ must be $G_{w}^{3}, G_{w}^{12}$ or, $G_{w}^{13}$, but $i_{+}\left(G_{w}^{12}\right)=i_{+}\left(G_{w}^{13}\right)=3$.

If $v(\mathcal{H}) \geq 3$, then by Lemma $2.4 i_{+}\left(G_{w}\right) \geq 3$ since $G_{w}$ contains $G_{w}^{12}$ or, $G_{w}^{13}$ as an induced subgraph.

Case 2. $\mathcal{H}$ has a $P_{2}$ as an induced subgraph.

If $\mathcal{H}=P_{2}, G_{w}$ must be $G_{w}^{4}$ or $G_{w}^{5}$. By Lemma $2.6, i_{+}\left(G_{w}^{4}\right)=i_{+}\left(G_{w}^{5}\right)=1+i_{+}\left(G_{w}^{\prime}\right)$, where $G_{w}^{\prime}$ is $\theta(1,1,1)_{w}$. By applying ECMOs on $A\left(G_{w}^{\prime}\right)$, we have $i_{+}\left(G_{w}^{4}\right)=i_{+}\left(G_{w}^{5}\right)=2$ if and only if the weight of $G_{w}$ satisfies the condition that $a_{2} b_{1}=a_{1} b_{2}$ and $a_{2} c_{1}=a_{1} c_{2}$.

If $\mathcal{H}$ contains the union of $P_{2}$ and an isolated vertex as an induced subgraph, then by Lemma $2.4, i_{+}\left(G_{w}\right) \geq 3$ since it contain one of $G_{w}^{i}$ 's $(i=14, \ldots, 19)$ as an induced subgraph.

If $\mathcal{H}$ contains a $P_{3}$ as an induced subgraph, then by Lemma $2.4, i_{+}\left(G_{w}\right)=1+i_{+}\left(G_{w}^{\prime}\right) \geq 3$, where $G_{w}^{\prime}$ is $G_{w}^{1}$ or $G_{w}^{2}$ and $i_{+}\left(G_{w}^{1}\right)=2, i_{+}\left(G_{w}^{2}\right) \geq 2$.

Next we characterize all graphs $G_{w}$ with $\theta(1,0,1)$ as its base satisfying $i_{+}\left(G_{w}\right)=2$ according to the following four possible cases.

Case 1. $\mathcal{H}$ is a collection of isolated vertices.

If $v(\mathcal{H})=1, G_{w}$ must be $G_{w}^{6}$ or $G_{w}^{7}$.

If $v(\mathcal{H})=2, G_{w}$ must be $G_{w}^{8}, G_{w}^{20}$ or $G_{w}^{21}$, but $i_{+}\left(G_{w}^{20}\right)=i_{+}\left(G_{w}^{21}\right)=3$.

If $v(\mathcal{H}) \geq 3$, then by Lemma $2.4, i_{+}\left(G_{w}\right) \geq 3$ since $G_{w}$ contains $G_{w}^{20}$ or $G_{w}^{21}$ as an induced subgraph.

Case 2. $\mathcal{H}$ is $P_{2}$. In this subcase, the underlying graph of $G_{w}$ must be $G_{w}^{9}$ or $G_{w}^{10}$, by calculation we have $i_{+}\left(G_{w}^{9}\right)=i_{+}\left(G_{w}^{10}\right)=2$ if and only if the weight of $G_{w}^{9}$ and $G_{w}^{10}$ satisfies the condition that $a_{2} c_{1}=a_{1} c_{2}$.

Case 3. $\mathcal{H}$ contains the union of $P_{2}$ and an isolated vertex as an induced subgraph. By Lemma $2.4, i_{+}\left(G_{w}\right) \geq 3$ since it contains one of $G_{w}^{i}$ 's $(i=22, \ldots, 27)$ as an induced subgraph.

Case 4. $\mathcal{H}$ contains a $P_{3}$ as an induced subgraph. By Lemma $2.4, i_{+}\left(G_{w}\right) \geq 3$ since $G_{w}$ contains $G_{w}^{28}$ or $G_{w}^{29}$ as an induced subgraph.

Now we characterize all graphs $G_{w}$ with $\theta(1,0,2)$ as its base satisfying $i_{+}\left(G_{w}\right)=2$ according to the following two possible cases.

Case 1. $\mathcal{H}$ is a collection of isolated vertices.

If $v(\mathcal{H})=1, G_{w}$ must be $G_{w}^{11}, G_{w}^{30}$ or $G_{w}^{31}$. Note that $i_{+}\left(G_{w}^{30}\right)=i_{+}\left(G_{w}^{31}\right)=3$, and by Lemmas 2.5 and 2.6, $i_{+}\left(G_{w}^{11}\right)=2$ if and only if the weight of $G_{w}^{11}$ satisfies $a_{1} a_{3}=a_{2} b_{1}$.

If $v(\mathcal{H}) \geq 2$, then by Lemma $2.4, i_{+}\left(G_{w}\right) \geq 3$ since $G_{w}$ contains $G_{w}^{30}$ or $G_{w}^{31}$ as an induced subgraph.

Case 2. $\mathcal{H}$ contains a $P_{2}$ as a induced subgraph, $G_{w}$ must be $G_{w}^{32}, G_{w}^{33}$ or $G_{w}^{34}$, but each of them have more than 2 positive eigenvalues.

At last, we consider graphs $G_{w}$ with $\theta(2,0,2)$ as its base satisfying $i_{+}\left(G_{w}\right)=2$. In fact, in this case, $G_{w}$ contains $G_{w}^{35}$ or $G_{w}^{36}$ as an induced subgraph.

Similarly, we can have the following theorems.

Theorem 4.5. Let $G_{w} \in \mathscr{B} \backslash \mathscr{B}_{p}$, then $i_{-}\left(G_{w}\right)=2$ if and only if $G_{w}$ is one of the following graphs: the weighted graph $\infty(4,1,4)_{w}$ with weighted condition $a_{1} a_{3}=a_{2} a_{4}$ and $b_{1} b_{3}=b_{2} b_{4}$; the weighted graph $\theta(1,1,1)_{w}$ with weighted condition $a_{2} b_{1} \neq a_{1} b_{2}$ or $a_{2} c_{1} \neq a_{1} c_{2}$; the weighted graph $\theta(1,0,1)_{w}$; the weighted graph $\theta(1,0,2)_{w}$ with weighted 
condition $a_{1} b_{2} \leq c_{1} c_{3}$; the weighted graph $\theta(2,0,2)_{w}$ with weighted condition $a_{2} b_{1} c_{3}-a_{1} a_{3} c_{2}-a_{2} c_{1} c_{3}=0$; the weighted graph $\theta(1,1,2)_{w}$ with weighted condition $a_{1} b_{2}=a_{2} b_{1}$.

Theorem 4.6. Let $G_{w} \in \mathscr{B}_{p}$ but no pendant twins, then $i_{-}\left(G_{w}\right)=2$ if and only if $G_{w}$ is one of the following graphs: the weighted graph $G_{w}^{1}, G_{w}^{3}, G_{w}^{6}, G_{w}^{8}$; the weighted graph $\left.G_{w}^{4}, G_{w}^{5}\right)$ with weighted condition $a_{2} b_{1}=a_{1} b_{2}$ and $a_{2} c_{1}=a_{1} c_{2}$; the weighted graph $G_{w}^{2}$ with weighted condition $a_{1} b_{2}=a_{2} b_{1}$; the weighted graph $G_{w}^{11}$ with weighted condition $a_{1} a_{3}=a_{2} b_{1}$.

\section{Weighted bicyclic graphs with rank $2,3,4$}

The rank of a weighted bicyclic graph $G_{w}$ is the rank of its adjacency matrix $A\left(G_{w}\right)$, denoted by $r\left(G_{w}\right)$. Then it is easy to see that $r\left(G_{w}\right)=i_{+}\left(G_{w}\right)+i_{-}\left(G_{w}\right)$. In this section, we'll characterize the weighted bicyclic graphs with rank $2,3,4$, respectively.

Theorem 5.1. Let $G_{w} \in \mathscr{B}$, then $r\left(G_{w}\right)=2$ if and only if $G_{w} \cong \theta(1,1,1)_{w}$ with weighted condition $a_{1} c_{2}=a_{2} c_{1}$ and $a_{1} b_{2}=a_{2} b_{1}$.

Proof. Let $G_{w}$ be a weighted bicyclic graph, $i_{+}\left(G_{w}\right) \geq 1$ and $i_{-}\left(G_{w}\right) \geq 1$ since $G$ contains $P_{2}$ as an induced subgraph. Then $r\left(G_{w}\right)=2$ if and only if $i_{+}\left(G_{w}\right)=i_{-}\left(G_{w}\right)=1$. By Theorems 4.1 and 4.2 we know $G_{w}$ must be $\theta(1,1,1)_{w}$ with weighted condition $a_{1} c_{2}=a_{2} c_{1}$ and $a_{2} b_{1}=a_{1} b_{2}$.

Theorem 5.2. Let $G_{w} \in \mathscr{B}$, then $r\left(G_{w}\right)=3$ if and only if $G_{w} \cong \theta(1,0,1)_{w}$ with weighted condition $a_{2} c_{1}=a_{1} c_{2}$.

Proof. Let $G_{w}$ be a weighted bicyclic graph, since $i_{+}\left(G_{w}\right) \geq 1$ and $i_{-}\left(G_{w}\right) \geq 1$, then $r\left(G_{w}\right)=3$ if and only if $i_{+}\left(G_{w}\right)=1, i_{-}\left(G_{w}\right)=2$ or $i_{+}\left(G_{w}\right)=2, i_{-}\left(G_{w}\right)=1$. Note that either $i_{+}\left(G_{w}\right)$ or $i_{-}\left(G_{w}\right)$ equals 1 , hence by Theorems 4.1 and 4.2 we know $G_{w}$ must be $\theta(1,0,1)_{w}$ with weighted condition $a_{2} c_{1}=a_{1} c_{2}$.

Theorem 5.3. Let $G_{w} \in \mathscr{B} \backslash \mathscr{B}_{p}$, then $r\left(G_{w}\right)=4$ if and only if $G_{w}$ is one of the following graphs: the weighted graph $\infty(4,1,4)_{w}$ satisfying $a_{1} a_{3}=a_{2} a_{4}$ and $b_{1} b_{3}=b_{2} b_{4}$; the weighted graph $\theta(1,1,1)_{w}$ satisfying $a_{2} b_{1} \neq a_{1} b_{2}$ or $a_{2} c_{1} \neq a_{1} c_{2}$; the weighted graph $\theta(1,0,1)_{w}$ satisfying $a_{2} c_{1} \neq a_{1} c_{2}$; the weighted graph $\theta(1,0,2)_{w}$ satisfying $a_{1} b_{2}=c_{1} c_{3}$; the weighted graph $\theta(2,0,2)_{w}$ satisfying $a_{2} b_{1} c_{3}-a_{1} a_{3} c_{2}-a_{2} c_{1} c_{3}=0$.

Proof. If $G_{w}$ be a weighted bicyclic graph, it is easy to know that $i_{+}\left(G_{w}\right) \geq 1$ and $i_{-}\left(G_{w}\right) \geq 1$. Then $r\left(G_{w}\right)=4$ if and only if $\left(i_{+}\left(G_{w}\right), i_{-}\left(G_{w}\right)\right)=(1,3)$ or $\left(i_{+}\left(G_{w}\right), i_{-}\left(G_{w}\right)\right)=(3,1)$ or $\left(i_{+}\left(G_{w}\right), i_{-}\left(G_{w}\right)\right)=(2,2)$. If one of $i_{+}\left(G_{w}\right)$ and $i_{-}\left(G_{w}\right)$ equals 1 , then $G_{w}$ must be $\theta(1,1,1)_{w}$ or $\theta(1,0,1)_{w}$, by Theorems 4.1 and 4.2 we know $r\left(G_{w}\right)<4$.

Hence, it suffices to consider that $\left(i_{+}\left(G_{w}\right), i_{-}\left(G_{w}\right)\right)=(2,2)$. By Theorems 4.3 and $4.5,\left(i_{+}\left(G_{w}\right), i_{-}\left(G_{w}\right)\right)=$ $(2,2)$ if and only if $G_{w}$ is one of the graphs described in Theorem 5.3.

Similarly, we can have the following theorem:

Theorem 5.4. Let $G_{w} \in \mathscr{B}_{p}$ but no pedant twins $n(n \geq 4)$, if $r\left(G_{w}\right)=4$ if and only if $G_{w}$ is one of the following graphs: the weighted graphs $G_{w}^{1}, G_{w}^{3}, G_{w}^{6}, G_{w}^{8}$; the weighted graph $G_{w}^{2}$ satisfying the weighted condition $a_{1} b_{2}=a_{2} b_{1}$; the weighted graph $G_{w}^{4}, G_{w}^{5}$ satisfying the weighted condition $a_{2} b_{1}=a_{1} b_{2}$ and $a_{2} c_{1}=a_{1} c_{2}$; the weighted graph $G_{w}^{11}$ satisfying the weighted condition $a_{1} a_{3}=a_{2} b_{1}$.

\section{References}

[1] M. Arav, F.J. Hall, Z.S. Li, H. van der Holst, The inertia set of a signed graph, Linear Algebra Appl. 439 (5) (2013) 1506-1529.

[2] L. Collatz, U. Sinogowitz, Spektren endlicher grapfen, Abh. Math. Sem. Univ. Hamburg 21 (1957) 63-77. 
[3] S. Daugherty, The inertia of unicyclic graphs and the implications for closed-shells, Linear Algebra Appl. 429 (2008) 849-858.

[4] Y.Z. Fan, W. Du, C. Dong, The nullity of bicyclic signed graphs, arXiv:1207.6765 [math.CO]

[5] Y.Z. Fan, Y. Wang, A note on the nullity of unicyclic signed graphs, Linear Algebra Appl. 397 (2005) $245-251$.

[6] Y.Z. Fan, K.S. Qian, On the nullity of bipartite graphs, Linear Algebra Appl. 430 (2009) 2943-2949.

[7] P.W. Fowler, D.E. Manolopoulos, An Atlas of Fullerenes, Clarendon Press, Oxford, 1995.

[8] D.A. Gregory, V.L. Watts, B.L. Shader, Biclique decompositions and Hermitian rank, Linear Algebra Appl. 292 (1999) 267-280.

[9] D.A. Gregory, B. Heyink, K.N. Vander Meulen, Inertia and biclique decompositions of joins of graphs, J. Comb. Theory Ser. B 88 (2003) 135-151.

[10] I. Gutman, N. Trinajstić, Graph theory and molecular orbitals, Top. Curr. Chem. 42 (1973) 49-93.

[11] S.B. Hu, B.L. Liu, X.Z. Tan, On the nullity of bicyclic graphs, Linear Algebra Appl. 429 (2008) 1387-1391.

[12] P. Lancaster, M. Tismenetsky, The Theory of Matrices, second ed., Academic Press Inc., Orlando, FL, 1985.

[13] S.C. Li, On the nullity of graphs with pendant vertices, Linear Algebra Appl. 429 (2008) 1619-1628.

[14] S.C. Li, F.F. Song, On the positive and negative inertia of weighted graphs, arXiv:1307.5110 [math.CO]

[15] H.C. Longuet-Higgins, Some studies in molecular orbital theory I. Resonance structures and molecular orbitals in unsaturated hydrocarbons, J. Chem. Phys. 18 (1950) 265-274.

[16] H.C. Ma, W.H. Yang, S.G. Li, Positive and negative inertia index of a graph, Linear Algebra Appl. 438 (1) (2013) 331-341.

[17] M. Nath, B.K. Sarma, On the null-spaces of acyclic and unicyclic singular graphs, Linear Algebra Appl. 427 (2007) 42-54.

[18] X.Z. Tan, B.L. Liu, On the nullity of unicyclic graphs, Linear Algebra Appl. 408 (2005) 212-220.

[19] G.H. Yu, L.H. Feng, Q.W. Wang, Bicyclic graphs with small positive index of inertia, Linear Algebra Appl. 438 (2013) 2036-2045. 